\title{
ENHANCING CONTEXT ANALYSIS WITH INTELLIGENCE IN PROVIDING Q-HEALTH SERVICES Less Infrastructure Dependency in Supporting Cardio-Vascular Patients
}

\author{
Boris Shishkov \\ IICREST / Department of Systems Engineering, TU Delft, Jaffalaan 5, Delft, The Netherlands \\ b.b.shishkov@iicrest.eu,b.b.shishkov@tudelft.nl \\ Alexander Verbraeck \\ Department of Systems Engineering, TU Delft, Jaffalaan 5, Delft, The Netherlands \\ a.verbraeck@tudelft.nl \\ Ing Widya \\ Department of Computer Science, University of Twente, Drienerlolaan 5, Enschede, The Netherlands \\ widya@ewi.utwente.nl
}

Keywords: Context-aware applications, Software components, e-Health services, Intelligent patient support.

Abstract: In Europe, we observe an increasing number of people with health problems, who could theoretically receive care outside of a hospital when their condition could be properly monitored. Not being able to provide this monitoring leads to an increasing pressure on an already overcrowded hospital system and increased costs. Ubiquitous technology on top of a high-quality IT infra-structure has already proven to be able to provide partial solutions. However, such infrastructure is not available throughout Europe. This brings the challenge of finding cheap, adaptive, and less infrastructure-dependent technological solutions. In the current paper we propose some solution ideas concerning the mentioned problems, which ideas are rooted in context analysis. In particular, we take context into account because adequately adapting the delivered services to the situation of the patient could result in more effective support. We incorporate multi-level context states and distinguish between some perspectives on context states for the design of flexible and adaptive ICT applications. The IT system autonomously considers context not only for the patient but also for the other related stakeholders such as caregivers. We illustrate our ideas with an example concerning cardio-vascular patients and expect the solutions to be extendable to other health care domains. The results can be generalized to also derive more fundamental knowledge on the possible ways in which context-aware solutions can be applied.

\section{INTRODUCTION}

In Europe, we observe an increasing number of people with health problems, who could theoretically receive care outside of a hospital when their condition could be properly monitored. Not being able to provide this monitoring leads to an increasing pressure on an already overcrowded hospital system and increased costs.

Ubiquitous technology on top of a high-quality IT infrastructure has already proven to be able to provide partial solutions. Tele-monitoring has been researched for years and the achieved results are more impressive than just hearing the heartbeat of a patient in Australia from The Netherlands, for example. There are more advanced things that could be done, such as the monitoring of epileptic patients at home and offering them immediate help when needed (A-MUSE Project, 2008), to mention just an example. These examples point, nevertheless, to multidisciplinary challenges, as they involve medical, logistical, and ICT issues. We approach the development of ICT applications from this multidisciplinary perspective, analyzing achieved results and proposing new solutions. A starting point we consider is appropriately analyzing context in 
developing applications and this concerns context modeling abstractions and a context handling platform. The context modeling abstractions provide application developers with proper conceptual foundations that can be extended and specialized with specific application requirements. The context handling platform allows some application functionality to be delegated to the platform, which reduces application development effort, time and, therefore, costs.

Until recently, computation was limited to an interaction style in which users provide to a desktop computer all the required input to perform particular tasks. Nowadays, computation is evolving to an interaction style in which explicit user intervention is gradually less required (Shishkov \& Van Sinderen, 2008). Explicit user inputs are being progressively replaced by conditions detected by sensors, devices and computers distributed in the environment. Hence, technological advances support (or enable) the shift of computing from the desktop paradigm into a paradigm in which computing is immersed in the dynamic world where we live and act (Moran \& Dourish, 2001). This paradigm shift poses many challenges, mainly related to whom, how, when and where to deliver services in the myriad of situations that can be encountered in the real world, which is the ever-changing context of use. In this view, capturing and monitoring the context in which services should be delivered, and adapting services according to the context and user preferences are essentially needed.

The underlying goal considered in this paper is finding cheap, adaptive, and less infrastructuredependent technological solutions in the abovediscussed perspective. We propose solution directions concerning these problems, which are rooted in context analysis. In particular, we take context into account because adequately adapting the delivered services to the situation of the patient could result in more effective support. We incorporate multi-level context states and distinguish between some perspectives on context states for the design of flexible and adaptive ICT applications. The IT system autonomously considers context not only for the patient but also for the other related stakeholders. We illustrate our ideas with an example concerning cardio-vascular patients and expect the solutions to be extendable to other health care domains. The results can be generalized to also derive more fundamental knowledge on the possible ways in which context-aware solutions can be applied.
With respect to the issue of providing support to cardio-vascular patients, it is to be noted that solutions to support the monitoring and help in case of emergency of patients require big investments and good IT infrastructure, which is often not the case in more isolated European countries, such as Bulgaria and Romania, while at the same time the severity of cardio-vascular problems is high there also. In these countries, some people with heart problems (often elderly people) who sometimes have nobody to take care of them, and who often suffer from lung and other problems (for instance, Chronic Obstructive Pulmonary Disease - COPD) at the same time, can rely on very limited support from the state, which makes it unrealistic to expect to apply advanced technology in the same way in which this is done in countries, such as Switzerland and The Netherlands. Therefore, innovative solutions building on a robust IT architecture are needed. For these particular problems, the use of context awareness is expected to provide the basis for a multidisciplinary solution.

The outline of this paper is as follows: Section 2 is furthering the discussion on context-awareness, including a detailed analysis and presenting our proposed views. Section 3 contains the running example that will be reflected in Section 4 in which we will partially illustrate our proposed views, elaborate more on our solution directions, and generalize the results accomplished. Finally, Section 5 contains the conclusions.

\section{ENHANCING CONTEXT ANALYSIS}

Context-aware applications are (in tune with the discussion so far) primarily motivated by their potential to increase user-perceived effectiveness, i.e. to provide services that better suit the needs of the end-user, by taking into account the user situation (Shishkov \& Van Sinderen, 2008). We refer to the collection of parameters that determine the situation of an end-user, and which are relevant for the application in pursue of user-perceived effectiveness, as end-user context, or context for short (as according to literature (Shishkov et al., 2008)). Context-awareness implies that information on the end-user context must be captured, preferably without conscious or active involvement of the enduser. Although in principle the end-user could also provide context information by directly interacting with the application, one can assume that in practice this would be too cumbersome if not impossible; it 
would require deep expertise to know the relevant context parameters and how these are correctly defined, and furthermore be very time consuming and error-prone to provide the parameter specifications as manual input. In case of health applications, context information is needed when end-users are least likely to be able to provide the input themselves. For these applications, it is even more important that the applications gather the context information independently of the end-user providing manual input. Context-aware applications can be particularly effective if the end-user is mobile and uses a personal handheld device for the delivery of services. The mobile case is characterized by dynamic context situations often dominated by changing location (however not necessarily restricted to this). Different locations may imply different social environments and different network access options, which offer opportunities for the provision of adaptive or value-added services based on context sensitivity. Especially in the mobile case, context changes are continuous, and a context-aware application may exploit this by providing near realtime context-based adaptation during a service delivery session with its end-user. The adaptation is 'near real-time' because context information is an approximation (not exact representation) of the reallife context and thus there may be a time delay. Through context-awareness, applications can be proactive with respect to service delivery, in addition to being just re-active, by detecting certain context situations that require or invite the delivery of useful services which are then initiated by the application instead of by a end-user request. Otherwise said, traditional applications provide service in reaction to user requests (re-active), whereas context-aware applications have also the possibility of initiating a service when a particular context situation is detected, without end-user input (pro-active). Although context-aware applications have received much attention within the research community, they have not been fully successful so far from a business point of view. This situation may change rapidly however, due to the observed growing power and reduced prices of mobile devices, sensors, and wireless networks, and due to the introduction of new marketing strategies and service delivery models (A-MUSE Project, 2008; Mei et al, 2007).

A context-aware application is (usually) expected to have the ability of adapting its service delivery depending on the information about the user's state (which information the application certainly must have received); this is illustrated in Figure 1.
As mentioned before, we are proposing a solution to some health-monitoring-related problems, which is rooted in context analysis. In user within
context

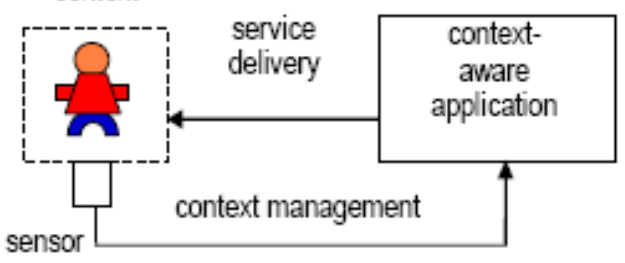

Figure 1: Schematic representation of a context-aware application (Shishkov \& Van Sinderen, 2008).

particular, we take context into account because adequately adapting the delivered services to the situation of the patient would result in more effective support. We incorporate multi-level context states and distinguish between different perspectives on context states for the design of flexible and adaptive ICT applications. The IT system autonomously considers context not only for the patient but also for the other related stakeholders.

We address hence these two innovative proposals, namely concerning: (i) multi-level context states and (ii) different perspectives on context states, in the remaining part of this section.

\subsection{Multi-level Context States}

In previous research (Shishkov et al, 2008; Shishkov \& Van Sinderen, 2008; A-MUSE Project, 2008), we have explored to some extent the possibilities to enforce context driven application adaptability, taking into account the situation of the end-user. What has not been exhaustively addressed nevertheless is how we take also into account the situations of other related entities. This we focus in the current paper. In particular, we suggest innovative ways of modeling context situations at different levels and we as well relate this to the mentioned already achieved results.

These different levels are needed because it is often not only the end-user whose situation is relevant. Taking for example the situation in which a patient is receiving support from a distance. We would often face the problem of considering other related entities as well - if the sensed vital signs of the patient indicate possible problems, it might be needed to provide careful analysis of a doctor, preferable the patient's own doctor, and if the situation gets worse, to send an ambulance to the patient. It has to be taken into account what the 
context situation of the doctor and the ambulance is. Maybe the ambulance of the local service point is in a traffic jam and it would be necessary to find another ambulance. It may be that the medical doctors in the emergency department of the hospital to which the patient is to be sent are busy with another emergency case and it would be wise sending the patient to another hospital in the area. Concluding, it can be stated that better care could be provided when a rich set of state information would be available, and could be combined appropriately for the particular case of the patient.

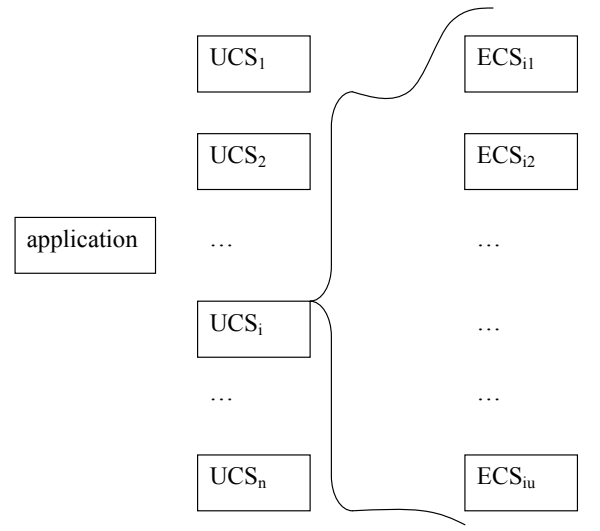

Figure 2: Considering many inter-dependent context situations.

Hence, we have (as Figure 2 suggests) a number of User Context States - UCS, based on which states the application should adapt its behavior, and each of these states points to a number of states of relevant entities.

We might like to further our previous work that puts all in a simpler setting where we abstract from the context situations of all other entities (ECS) which situations would be relevant to the desirable application service delivery.

\subsection{Different Perspectives on Context States}

Monitoring of patients remotely, especially for disease management or supervision of elderly persons with systemic diseases, is often a multifacetted process. The current perspective of the World Health Organization (WHO) on health is in fact extended from a bio-psycho health model, typically expressed in terms of body functional and structural impairments and disabilities, to a biopsycho socio model, additionally expressed with terms of person's participation in the society (WHO, 2001). This vision on health and the current trends for patient self-management require orchestrated multi-level loops of monitoring and treatment controls because of the different dynamic properties that concern the management of the systemic diseases and the person's participation.

A patient suffering from a chronic cardiovascular disease, for example, may need continuous monitoring of his or her heart condition. The dynamics of the monitoring or treatment of this patient would often depend on the selected post diagnostic treatment protocol (Von Wright, 1963). In case of COPD as well, there would be an additional disease management protocol needed, and it would be of course necessary to appropriately align this protocol with the (primary) cardiovascular disease management protocol.

The ICT facilities that should support the patient are to be configured therefore in accordance with the different disease management dynamics. This would involve in turn several context state trajectories, each of which reflecting the particular disease management protocol. Consequently, the patient supervision processes would also require several context state trajectories, each one in conformity with the dynamics of the specific disease management protocol (Moran \& Dourish, 2001; Meinert, 1986).

Besides these multiple bio-medical context state trajectories, another context state trajectory is needed in order to reflect the supervision of the participation of the patient. Such supervision may provide a better feeling of safety and availability of on demand assistance; these often lower the thresholds of participation. State changes of the patient's external environment are typically slow; this is in contrast to emergency care or some warning events in chronic care. Accordingly, other control or management techniques may apply for monitoring of patients to improve participation.

Hence, treating the discussed context state trajectories separately, while maintaining coordination at the same time, is believed to be a better design practice of separation of concerns.

In the following section - Case Example, we present a scenario that illustrates a contextual setting for patient monitoring to improve patient's participation.

\section{CASE EXAMPLE}

As mentioned already, we will present in this section a case example through which we will illustrate our ideas. 
Our scenario envisions Rob Martens who is 70 years of age; he lives in a service apartment complex for elderly. Being diagnosed as a heart patient, he participates in a cardio-vascular monitoring program of the local hospital's Cardiology Department. As many others of his age with a heart problem, Rob also suffers from COPD. Although Rob can live on his own, he often gets out of breath and falls if not being able to find out a nearby place to sit.

For the monitoring program, Rob wears a garment with dry ECG electrodes, a 3D acceleration activity sensor with GPS and as well a PDA which collects, processes, multiplexes and transfers Rob's vital signs continuously and wirelessly to the Cardio-Care portal of the Cardiology Department. A specialized nurse continuously occupies this portal and a junior cardiologist is always on duty at the department.

Being reluctant (before participating in the monitoring program) to go out of his apartment for socialization and even preferring to stay in his bedroom (because of his feeling insecure), Rob feels more secure when he knows that they continuously monitor him and he goes down often, visiting the common room in the complex.

On Day X, Rob goes early to the common room, but after a few hours he feels tired and he walks back to his apartment. In the elevator, Rob feels dizzy and falls down. He does not manage to sit on the bench in the elevator, neither to reach the elevator's alarm button. Rob's sensory system detects the fall however; the system is aware that he is in the elevator and thus this is known at the Cardio-Care portal. The portal also detects an increase of Rob's heart rate, it beeps for the attention of the nurse on duty, Marit, renders Rob's data from his electronic patient record and switches on the audio-visual system (display, speakers, microphone and remotely controllable camera) in the elevator that is usually switched off for privacy and energy saving reasons. Via the Hi-Fi audio link Marit calms Rob down, which effect she observes from his heart rate reading. The trained nurse concludes from the vital sign readings and the camera display on her monitor that Rob's condition is not acute and decides therefore not to ask for an ambulance and not to reset the elevator to go to the ground floor, Instead, Marit clicks on her screen for a window presenting the nurses in the vicinity of Rob including their prioritized activity from their electronic work schedule and their ability to assist Rob, taking into consideration that Rob is too heavy for many of the nurses. Marit only detect Dewi at a floor higher and who needs a lift device to help Rob.
Marit reset the lift to go a floor higher and beeps Dewi, who finds immediately the location of the nearest lift device since all devices have RFD tags, and who thus can help Rob to go back to his apartment. In the mean time, Marit sends a report containing the vital sign readings, the video clip and the symptoms to John, a Cardio-Care nurse practitioner who is assigned to Rob.

In the following section, we will further elaborate our ideas, reflecting them in the partial application of this case scenario.

\section{SUPPORTING CARDIO-VASCULAR PATIENTS INTELLIGENTLY}

We will analyse the scenario presented in the previous section, in a practical application, and while doing this, we will elaborate our innovative ideas, that have already been presented in Section 2 .

Logically, the identification of the domainimposed requirements should be firstly done. The entities and some of their activities, in a typical scenario, are represented in Figure 3.

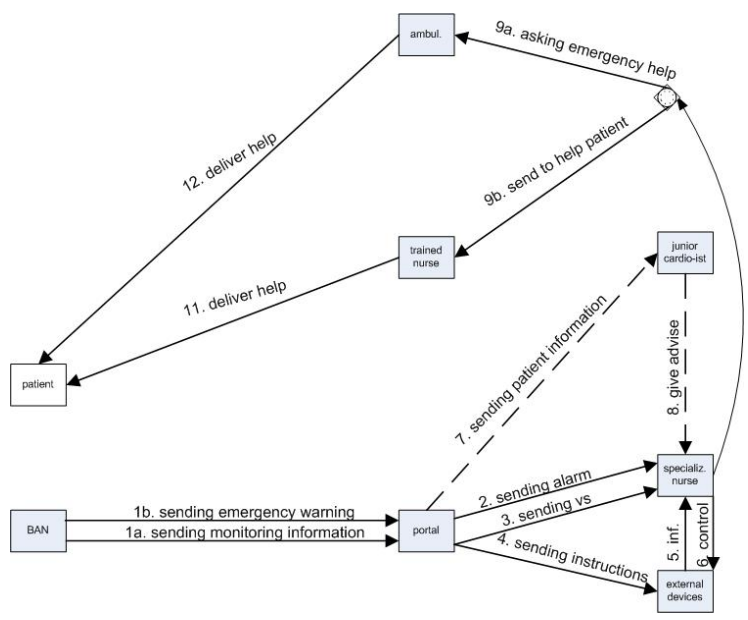

Figure 3: Entities and activities in a typical scenario.

In the Figure, the BAN (Body Area Network) is associated with the Patient but it is another entity because it is not the patient who controls the BAN sensors but they are performing their tasks on their own, together with the BAN intelligent devices. The BAN usually sends monitoring information to the Portal (1a) and in cases of a (sensed) emergency it can also send an emergency warning (1b). We will consider only the emergency case further. Hence, following the warning (1b), the Portal should send 
an alarm to a specialized nurse (2) and it should transmit the sensor readings (3). At the same time, instructions could be given to external devices, such as cameras in elevators, for instance. This triggers the devices to send some actual information to the specialized nurse (5) who can manage in turn to control these devices for even better results (6). Sometimes, when the case appears to be of great complexity, the specialized nurse may ask a junior cardiologist to get involved in the decision making (8), supported by information concerning the patient, which can be received from the Portal (7). Based on all the information and the potential input of the junior cardiologist, the specialized nurse should decide whether the situation is urgent or not. If not, then a trained nurse is to be asked to deliver help (9b). In case of emergency nevertheless, the specialized nurse must ask help from an ambulance (9a). No matter what the choice, the patient is to receive help - either from the trained nurse (11) or from the emergency team (12).

This information can be reflected usefully in other models, as according to the SDBC approach (Shishkov, 2005).
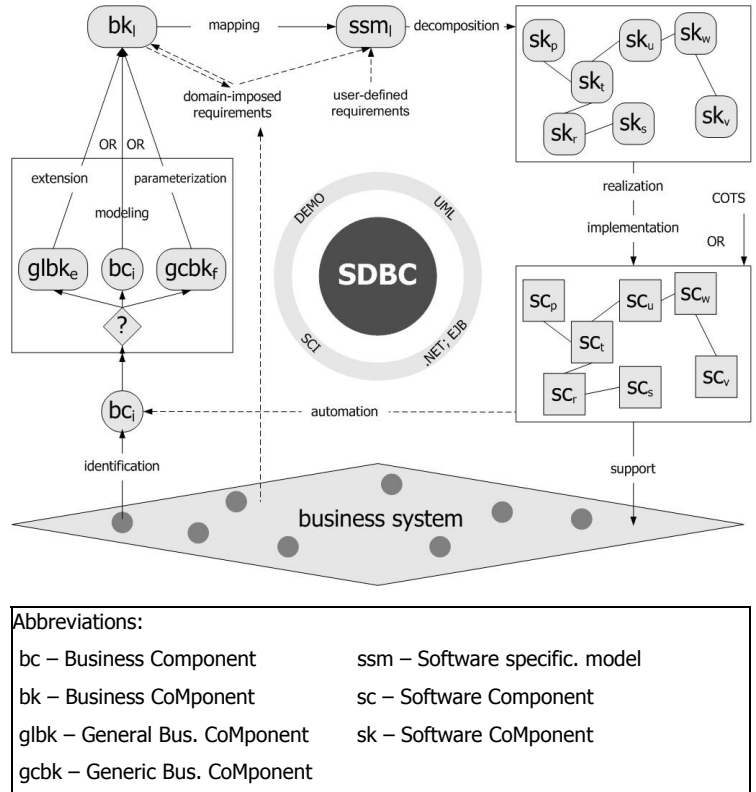

Figure 4: SDBC Approach (Shishkov, 2005).

As presented in Fig. 4, we consider a Business System (with a static perspective and a dynamic perspective, which both can be projected on the model we have presented in Figure 3; we omit a discussion on these modeling transformations for reasons of brevity) from which Business Components are to be identified (these are just parts of the Business System). The component should be then reflected in a relevant model - a Business CoMponent (these are business models (Van Hee \& Reijers, 2000) that are to be used as foundation in deriving the specification of an automated system). Another way for arriving at a Business CoMponent is through re-use, which we also omit for brevity. The Business CoMponents should then be elaborated with more domain-imposed requirements, in order to add elicitation on the particular context in which its corresponding Business Component exist within the Business System, and these are elaborations added to the relationships expressed in Figure 3. Then, a mapping towards a software specification model should take place (Rational: OMG, 2007), through a modeling transformation, taking into account that all that is not to be automated appears as the environment of the ICT system (Liu, 2000) and all that affects its functionality - as context.

We are therefore able to define generic ICT components that are adjustable based on the particular situation in the business world. These could be used in the specification of context-aware applications that effectively support patients. The component-based approach has a crucial advantage with respect to the service-orientation since technical components and corresponding business services are to be considered in concert, setting up an orchestration of the services with the users (not burdened with technology) and adequately tracing to the technology, keeping track also which part of the delivered functionality is the responsibility of an application component and which part is the responsibility of the underlying infrastructure.

On the basis of this study, we claim that components are a good foundation for serviceorientation and that service-orientation in turn is a good foundation for context awareness (because context awareness means different functionality versions, which naturally projects to different related services). All this in combination can be of particular value, nevertheless if only the real-life information is adequately captured and processed, as we have suggested in this section.

\section{CONCLUSIONS}

In this paper, we have reported follow-up research from previous studies on context-aware application support to patients, mainly envisioning telemonitoring. We have added contribution on how to better describe, present and capture the real-life 
information and reflect it in software components in a consistent way.

We have omitted for brevity discussions on modeling transformations that have been considered exhaustively in literature, especially in materials focusing on model-driven engineering. What is missing in most of these materials nevertheless is sound guidelines on the identification of computation-independent models and in the paper we have delivered some useful contribution relevant to this. Furthermore, we have put some concreticity with regard to more general results from previous studies, considering especially the health care perspective where context-awareness is of crucial importance. Finally, we have also illustrated partially the early stages of deriving a computationindependent model, by emphasizing on the need for structuring information as good as possible.

In the analysis preceding the proposed methodological guidelines, we have suggested how multi-level context states can be incorporated and how context states can be considered from different perspectives, for the design of flexible and adaptive ICT applications.

The achieved results can be generalized to also derive more fundamental knowledge on the possible ways in which context-aware solutions can be applied.

We plan for further research reflecting the mechanisms of the SDBC approach for structuring early requirements in a separate methodology, applied in different domains including the healthcare domain.

\section{REFERENCES}

A-MUSE Project, 2008: http://a-muse.freeband.nl.

Liu, K., 2000. Semiotics in Information Systems Engineering, Cambridge University Press. Cambridge.

Mei, H., Widya, I.A., Broens, T.H.F., Pawar, P., Van Halteren, A.T., Shishkov, B., Van Sinderen, M.J., 2007. A Framework for Smart Distribution of Biosignal Processing Units in m-Health. In ICSOFT'07, 2nd International Conference on Software and Data Technologies. INSTICC Press.

Meinert, C.L., 1986. Clinical Trial: Design, Conduct and Analysis, Oxford University Press, New York.

Moran, T.P. and Dourish, P, 2001. Introduction to Context-Aware Computing, Special Issue of HumanComputer Interaction, Volume 16, IBM Almaden Research Center, University of California, Irvine.

Rational / OMG UML, 2007. Unified Modeling Language, Object Management Group, http://www.omg.org/uml.
Shishkov, B., 2005. Software Specification Based on Reusable Business Components, Delft University Press, Delft ( $\mathrm{PhD}$ Thesis).

Shishkov, B., Van Sinderen, M.J., Liu, K., Du, H., 2008. Norm Analysis Supporting the Design of ContextAware Applications. In ICEIS'08, 10th International Conference on Enterprise Information Systems. INSTICC Press.

Shishkov, B. and Van Sinderen, M.J., 2008. On the Design of Context-Aware Applications. In I-WEST'08, 2nd International Workshop on Enterprise Systems and Technology. INSTICC Press.

Van Hee, K. and Reijers, H.A., 2000. Using Formal Analysis Techniques in Business Process Re-Design. W. van der Aalst et al. (Eds.): Business Process Management, LNCS 1806.

Von Wright, G. H., 1963. Norms and Action - a Logical Enquiry. Routledge and Kegan Paul, New York.

World Health Organization - WHO. 2001. International Classification of Functioning, Disability and Health: ICF. Geneva, Switzerland. 